OPEN ACCESS

Edited by:

Yuji Morita,

Aichi Gakuin University, Japan

Reviewed by:

Xian-Zhi Li,

Health Canada, Canada José Manuel Rodriguez-Martínez,

University of Seville, Spain

Veronique Dubois,

University of Bordeaux, France

*Correspondence:

Ashima K. Bhardwaj

ashima.bhardwaj@gmail.com

Specialty section: This article was submitted to Antimicrobials, Resistance

and Chemotherapy,

a section of the journal

Frontiers in Microbiology

Received: 14 September 2015

Accepted: 26 January 2016

Published: 15 February 2016

Citation:

Vinothkumar K, Kumar GN

and Bhardwaj AK (2016)

Characterization of Vibrio fluvialis

anrVC5 Gene in Native

and Heterologous Hosts: Synergy of anrVC5 with other Determinants in Conferring Quinolone Resistance.

Front. Microbiol. 7:146.

doi: 10.3389/fmicb.2016.00146

\section{Characterization of Vibrio fluvialis qnrVC5 Gene in Native and Heterologous Hosts: Synergy of qnrVC5 with other Determinants in Conferring Quinolone Resistance}

\author{
Kittappa Vinothkumar ${ }^{1,2}$, G. N. Kumar ${ }^{2}$ and Ashima K. Bhardwaj ${ }^{\text {* }}$ \\ ${ }^{1}$ Molecular Biology of Diseases, Department of Human Health and Diseases, School of Biological Sciences and \\ Biotechnology, Indian Institute of Advanced Research, Gandhinagar, India, ${ }^{2}$ Department of Bio-Chemistry, Faculty of \\ Science, The Maharaja Sayajirao University of Baroda, Vadodara, India
}

Resistance of various pathogens toward quinolones has emerged as a serious threat to combat infections. Analysis of plethora of genes and resistance mechanisms associated with quinolone resistance reveals chromosome-borne and transferable determinants. qnr genes have been found to be responsible for transferable quinolone resistance. In the present work, a new allele qnrVC5 earlier reported in Vibrio fluvialis from this laboratory was characterized in detail for its sequence, genetic context and propensity to decrease the susceptibility for quinolones. The study has revealed persistence of qnrVC5 in clinical isolates of $V$. fluvialis from Kolkata region through the years 20022006. qnrVC5 existed in the form of a gene cassette with the open reading frame being flanked by an upstream promoter and a downstream $V$. cholerae repeat region suggestive of its superintegron origin. Sequence analysis of different qnrVC alleles showed that qurVC5 was closely related to qnrVC2 and qnrVC4 and these alleles were associated with $V$. cholerae repeats. In contrast, qnrVC1, qnrVC3, and qnrVC6 belonging to another group were associated with $V$. parahaemolyticus repeats. The gene manifested its activity in native $V$. fluvialis host as well as in Escherichia coli transformants harboring it by elevating the MIC toward various quinolones by twofold to eightfold. In combination with other quinolone resistance factors such as topoisomerase mutations and $\mathrm{aac}\left(6^{\prime}\right)-\mathrm{Ib}-\mathrm{Cr}$ gene, qnrVC5 gene product contributed toward higher quinolone resistance displayed by $V$. fluvialis isolates. Silencing of the gene using antisense peptide nucleic acid sensitized the $V$. fluvialis parent isolates toward ciprofloxacin. Recombinant QnrVC5 vividly demonstrated its role in conferring quinolone resistance. qnrVC5 gene, its synergistic effect and global dissemination should be perceived as a menace for quinolone-based therapies.

Keywords: gene cassettes, plasmids, superintegron, quinolone susceptibility 


\section{INTRODUCTION}

Vibrio fluvialis is known to cause severe cholera-like diarrhea in humans and has been perceived as an emerging pathogen (Bhattacharjee et al., 2010; Ramamurthy et al., 2014). Diarrheal illnesses caused by this kind of bacteria are generally treated using quinolone class of antibiotics. Increase in the reports of emergence of multi drug resistant (MDR) V. fluvialis, showing considerable resistance to quinolones has been a public health concern (Srinivasan et al., 2006; Singh et al., 2012; Ramamurthy et al., 2014). Reduced susceptibility to quinolones in bacteria is mediated by factors such as mutations in the genes for DNA gyrase and topoisomerase IV (the drug targets), efflux activity, Qnr proteins and the inactivation of drug by quinolonemodifying enzyme [AAC (6') Ib-Cr; Bhardwaj et al., 2014; Kim and Hooper, 2014]. The above mentioned mechanisms may work alone or in combination. The synergistic action of these mechanisms helps the pathogen to achieve higherlevel of resistance toward quinolones (Baranwal et al., 2002; Srinivasan et al., 2006; Rushdy et al., 2013; Zhu et al., 2013).

Qnr proteins are pentapeptide repeat proteins (PRPs) which protect DNA gyrase from quinolone action (Tran and Jacoby, 2002). qnr, a plasmid-mediated horizontally transferable gene conferring quinolone resistance, was first discovered in a plasmid from Klebsiella pneumoniae in 1998 (MartinezMartinez et al., 1998). Thereon, different variants of $q n r$ genes such as $q n r A, q n r B, q n r C, q n r D$, and $q n r S$ were reported in different pathogens from different parts of the world (Strahilevitz et al., 2009). Chromosomal qnr-like genes such as $q n r V C, \mathrm{Ppqnr}, \mathrm{Vpqnr}$, and Smqnr were also reported from $V$. cholerae (Fonseca et al., 2008), Photobacterium profundum (Poirel et al., 2005a), V. parahaemolyticus (Saga et al., 2005), and Stenotrophomonas maltophilia (Sanchez et al., 2008), respectively. Qnr proteins cause low-level resistance to quinolones, facilitating the emergence of resistant mutants. In combination with other mechanisms of quinolone resistance such as topoisomerase mutations and efflux action, Qnr proteins can help the pathogens to achieve clinical breakpoints of quinolone resistance (Martinez-Martinez et al., 2003; Jeong et al., 2008).

Till date, structures for PRPs such as MfpA, EfsQnr, QnrB1, and AhQnr have been solved (Hegde et al., 2005, 2011; Vetting et al., 2011; Xiong et al., 2011). QnrB1 (from K. pneumoniae) and AhQnr (from Aeromonas hydrophila) possessed a right handed quadrilateral $\beta$-helix, which is typical of PRPs and encompassed coils with two loops (loop A and loop B) extending outward from the regular structure, interrupting the $\beta$-helix turn. Unlike the PRP structures of MfpA and EfsQnr from Gram-positive bacteria Mycobacterium tuberculosis and Enterococcus faecalis, respectively, QnrB1 and AhQnr possessed these two loops. It was established that these two loops played a vital role in the interaction of Qnr and DNA gyrase subunits (GyrA and GyrB; Xiong et al., 2011). Loop A was found to interact with GyrA "tower" whereas loop B was found to interact with GyrB TOPRIM (Topoisomerase-Primase) domains. This also indicated that the mechanisms of interaction of MfpA and EfsQnr with DNA gyrase were different from that of QnrB1 and AhQnr. These loops are found to be conserved among all plasmid-based Qnr variants and some chromosome-borne Qnr proteins (Vetting et al., 2011).

A former belief based on the mode of action of MfpA and EfsQnr explains that Qnr protein binds with DNA gyrase and prevents the formation of cleaved complex. Qnr protein does not interact with the quinolones and therefore renders resistance to drugs indirectly (Hegde et al., 2005, 2011). As the structure of MfpA and EfsQnr varied from QnrB1 and AhQnr, their mode of action should also principally vary. The model proposed by Vetting et al. suggested that QnrB1 protein binds to and destabilizes the topoisomerase-quinolone-DNA cleavage complex, which eventually results in the release of quinolone and religation of DNA. After this process, the Qnr protein would be released and the active form of topoisomerase would be regenerated (Vetting et al., 2011).

Emergence of transferable qnrVC alleles in Vibrionaceae family and other bacterial species aggravated the hysteria on quinolone resistance (Fonseca and Vicente, 2013; Pons et al., 2013). So far, seven $q n r V C$ alleles (named as $q n r V C 1$ to $q n r V C 7$ ) have been reported from different parts of the globe ${ }^{1}$. These alleles are found as gene cassettes, equipped with all the elements necessary for their mobility, incorporation and expression such as attC sites and their own cassette-specific promoter (Fonseca and Vicente, 2013).

Previous studies from this laboratory have revealed the presence of a qnrVC-like gene in the plasmids of three $V$. fluvialis clinical isolates, BD146, L10734, and L9978 (Rajpara et al., 2009; Singh et al., 2012) and this allele was named qnrVC5 by Fonseca and Vicente in 2013 (Fonseca and Vicente, 2013). The plasmid that harbored qnrVC5 in V. fluvialis BD146 also carried the gene encoding trimethoprim resistance $(d f r V I)$ and showed 99\% identity with pVN84 from $V$. cholerae O1 isolated from Vietnam (Rajpara et al., 2009) and plasmid from V.parahaemolyticus V110 isolated from Hong Kong, China (Liu and Chen, 2013; Bhardwaj, 2015). The plasmid from $V$. parahaemolyticus V110 possessed $q n r V C 5$ allele whereas plasmid pVN84 carried qnrVC2, a nonfunctional form of $q n r V C 5$ due to the presence of several internal stop codons (Fonseca et al., 2008; Fonseca and Vicente, 2013). From the above discussion and other reports, it is amply clear that $q n r V C$ genes are disseminated globally and are likely to play a vital role in quinolone resistance due to their wide dispersal (Rajpara et al., 2009; Kim et al., 2010; Kumar and Thomas, 2011; Fonseca and Vicente, 2013; Liu and Chen, 2013; Pons et al., 2013; Bhardwaj, 2015). Since qnrVC5 allele was first reported from this laboratory, it was of prime interest to decipher its role in conferring resistance to quinolones. Therefore, the present study was undertaken to understand the features of this gene/its product in silico. Another focus of the study was to functionally characterize QnrVC5 protein in native $V$. fluvialis host and heterologous E. coli host. Results reflected the role of qnrVC5 in conferring resistance to quinolones and its synergy with other quinolone resistance factors such as mutations in topoisomerase genes and $\operatorname{aac}\left(6^{\prime}\right) \mathrm{Ib}$-cr gene.

\footnotetext{
${ }^{1}$ http://www.lahey.org/qnrstudies/
} 


\section{MATERIALS AND METHODS}

\section{Bacterial Strains and Plasmids}

Clinical isolates of V. fluvialis BD146, L13828, L10734, L9978, and L15318 were obtained from patients suffering from acute cholera-like diarrhea admitted to The Infectious Diseases Hospital, Kolkata during 2002-2006 (Kind gift from Dr. T. Ramamurthy, National Institute of Cholera and Enteric Diseases, Kolkata), and have been used in earlier studies (Rajpara et al., 2009; Singh et al., 2012). Plasmid pET 28a (Novagen) was used for expression of qnrVC5 gene. E. coli JM109 was used for transformation experiments and E. coli BL21 ( $\lambda$ DE3) was used for recombinant protein expression studies.

\section{In Silico Analysis}

The DNA sequence analyses were done using BLAST tool available at NCBI $s^{2} e^{2}$ and phylogenetic tree was constructed using maximum-likelihood method in MEGA6 (Tamura et al., 2013). Softberry-BPROM, a promoter prediction tool was used to find the promoters and other regulatory elements in qnrVC5 gene cassette $^{3}$. The structure of QnrVC5 was predicted by I-TASSER server using automated mode, as it employs hierarchical method for protein structure and function prediction using multiple threading approach based on structural templates from PDB (Zhang, 2008; Roy et al., 2010, 2012).

\section{Peptide Nucleic Acid (PNA)}

The anti-qnrVC peptide-PNA $\left[\mathrm{H}-(\mathrm{KFF})_{3} \mathrm{~K}-\mathrm{O}\right.$-ccattttctagccct$\mathrm{NH}_{2}$ ] complementary to the region encompassing ribosomal binding site and start codon of qnrVC5 gene was designed to silence the qnrVC5 gene at RNA level. The PNA was conjugated with cell penetrating peptide $(\mathrm{KFF})_{3}$ to enhance the permeability of this antisense oligonucleotide across the cell membrane of bacteria (Good et al., 2001). The peptide-PNA was synthesized by PANAGENE (Daejeon, South Korea) and the lyophilized PNA oligomer was dissolved in sterile water as per manufacturer's instructions.

\section{DNA Preparations}

The preparations of genomic and plasmid DNA of $V$. fluvialis were done as described earlier (Thungapathra et al., 2002). Plasmid Mini kit or Maxi kit (Qiagen) was used for plasmid DNA isolation according to the manufacturer's instructions.

\section{Polymerase Chain Reaction (PCR) and Reverse Transcription PCR (RT-PCR)}

Genomic DNA (200 ng) or plasmid DNA (10-50 ng) were used as templates for PCR reactions. PCR reactions were performed using the protocol described earlier (Singh et al., 2012). Primers used for PCR experiments were qnrVC-F $5^{\prime}$-CGCGGATCCATGGATAAAACAGACCAG-3' and qnrVCR $\quad 5^{\prime}$-CCGCTCGAGTTAGTCAGGAACTACTAT- $3^{\prime}$. These

\footnotetext{
${ }^{2}$ http://blast.ncbi.nlm.nih.gov/Blast.cgi

${ }^{3} \mathrm{http}: / /$ linux 1 .softberry.com/berry.phtml?topic=bprom\&group=programs\& subgroup $=$ gfindb
}

primers incorporated sites (in bold) for BamHI and XhoI restriction enzymes that were used for cloning in the expression vector pET28a. Primers used to amplify the entire qnrVC5 gene cassette, encompassing the upstream and downstream sequences along with coding region, were qnrVCcas-F $5^{\prime}$-CGTATAGAAAGCGTTATGTG- $3^{\prime}$ and qnrVCcas-R $5^{\prime}$ CTGCTGCCATAATGGATAT- $3^{\prime}$. Each PCR consisted of an initial denaturation at $94^{\circ} \mathrm{C}$ for $5 \mathrm{~min}$, followed by 30 amplification cycles, each involving an initial denaturation at $94^{\circ} \mathrm{C}$ for $0.5 \mathrm{~min}$ followed by annealing and extension steps. Annealing condition for qnrVC-F and qnrVC-R primer pair was $65^{\circ} \mathrm{C}$ for $0.5 \mathrm{~min}$, and for qnrVCcas- $\mathrm{F}$ and qnrVCcas- $\mathrm{R}$ primer pair was $60^{\circ} \mathrm{C}$ for $0.5 \mathrm{~min}$. For both the above reactions, extension was performed at $72^{\circ} \mathrm{C}$ for $1 \mathrm{~min}$ and final polymerization was carried out at $72^{\circ} \mathrm{C}$ for $10 \mathrm{~min}$. Taq polymerase (Fermentas) was used and reactions were performed in T100 thermal cycler (Bio-Rad Laboratories). Purification of PCR products was performed using QIA-quick PCR purification kit (Qiagen) as per the manufacturer's instructions.

RT-PCR was carried out to confirm the expression of qnrVC5 gene and $a a c\left(6^{\prime}\right) \mathrm{Ib}$-cr gene in the native isolates of $V$. fluvialis and their E. coli JM109 transformants. Total RNA was isolated from $V$. fluvialis isolates and their E. coli transformants, using RNeasy bacteria mini kit (Qiagen) as per the manufacturer's instructions. The protocol consisted of growing the cultures in LB medium followed by treatment with lysozyme and proteinase $\mathrm{K}$ for cell lysis and RNA was purified from the lysate using RNeasy mini spin columns. The RNA preparations were subsequently treated with DNaseI (Fermentas) to remove the genomic DNA contamination. RT-PCR was carried out using Qiagen one step RT-PCR kit following manufacturer's instructions. Each RT-PCR reaction mixture consisted of $10 \mu \mathrm{l}$ of $5 \mathrm{X}$ Qiagen 1-step RTPCR buffer, $2.0 \mu \mathrm{l}$ of dNTP mix containing $2.5 \mathrm{mM}$ of each dNTP, $50.0 \mathrm{pmol}$ of each primer, $2.0 \mu \mathrm{l}$ of Qiagen 1-step RT-PCR enzyme mix and RNase free water to a final volume of $49.0 \mu \mathrm{l}$. The $1.0 \mu \mathrm{l}$ template RNA $\left(0.1 \mu \mathrm{g} \mu \mathrm{l}^{-1}\right)$ was added to make the final reaction volume of $50.0 \mu \mathrm{l}$. The primer pair qnrVC-F and qnrVC-R, mentioned above, were used for RT-PCR of qnrVC5 transcripts, whereas AG-F 5' - TGACCAACTGCAACGATTCC $3^{\prime}$ and AG-R 5' - ACCCATAGAGCATCGCAAGGT - $3^{\prime}$ were used for RT-PCR of $a a c\left(6^{\prime}\right) I b-c r$ transcripts. Each RT-PCR experiment consisted of reverse transcription step at $50^{\circ} \mathrm{C}$ for $30 \mathrm{~min}$ and an initial denaturation at $95^{\circ} \mathrm{C}$ for $15 \mathrm{~min}$, followed by 30 amplification cycles, each consisting of a denaturation step at $94^{\circ} \mathrm{C}$ for $0.5 \mathrm{~min}$ followed by annealing and extension steps. The annealing condition for qnrVC5 amplification was $60^{\circ} \mathrm{C}$ for $0.5 \mathrm{~min}$ and for $a a c\left(6^{\prime}\right) \mathrm{Ib}$-cr amplification was $64^{\circ} \mathrm{C}$ for $0.5 \mathrm{~min}$. Extension was performed at $72^{\circ} \mathrm{C}$ for $1 \mathrm{~min}$ and final polymerization was carried out at $72^{\circ} \mathrm{C}$ for $10 \mathrm{~min}$. The reactions were performed in T100 thermal cycler (Bio-Rad Laboratories).

\section{Gene Cloning and Protein Expression}

Vector pET28a and the amplicon of qnrVC5 (from $V$. fluvialis BD146) each were separately double-digested with BamHI and XhoI. Subsequent to digestion, the dephosphorylated vector and qnrVC5 insert were ligated, the ligation mixture was electroporated in E. coli JM109 cells and the transformed cells 
were plated on LB plates containing kanamycin $\left(50 \mu \mathrm{g} \mathrm{ml}^{-1}\right)$ to obtain the recombinants. Expression of QnrVC5 protein was studied by induction of $E$. coli BL21 ( $\lambda \mathrm{DE} 3$ ) cells harboring the recombinant clones with $1 \mathrm{mM}$ IPTG for $2 \mathrm{~h}$ at $37^{\circ} \mathrm{C}$, followed by SDS-PAGE analysis of total cell lysates. The protein band corresponding to QnrVC5 was excised from the gel and subjected to trypsin digestion. The authenticity of this protein was then confirmed by peptide mass fingerprinting on Bruker Ultraflex III MALDI instrument. The protein identification was done through Mascot software.

\section{Minimum Inhibitory Concentration (MIC) Assays}

Twofold dilution method was used to determine the MIC of various quinolones for native $V$. fluvialis clinical isolates, E. coli transformants and QnrVC5 recombinant as described previously with minor modifications described below (Mohanty et al., 2012).

(i) MIC assay for native $V$. fluvialis clinical isolates and their E. coli JM109 transformants

Overnight grown colonies of $V$. fluvialis were inoculated in $5 \mathrm{ml} \mathrm{LB}$ and grown at $37^{\circ} \mathrm{C}$ till the optical density at $600 \mathrm{~nm}$ reached 0.1 . This culture was used as inoculum for MIC assay. Concentration of the test drug was diluted twofold in Muller Hinton Broth (MHB). Fifty microliters of inoculum was added to $2 \mathrm{ml}$ of each drug concentration in a 24-well cell culture plate and incubated at $37^{\circ} \mathrm{C}$ for $18 \mathrm{~h}$. MIC was read as the lowest concentration of the drug where no growth was observed. The assays were repeated at least three times. The MIC of E. coli JM109 transformants (obtained from the plasmid preparation derived from the native $V$. fluvialis host) was determined by the same method except that the transformants were selected in LB medium containing ampicillin $\left(25 \mu \mathrm{g} \mathrm{ml}^{-1}\right)$ before inoculating into the MIC assay plate.

\section{(ii) MIC assay for QnrVC5 recombinants}

The recombinant plasmid was transformed into E. coli BL21 $(\lambda \mathrm{DE} 3)$ cells and the transformants were selected on LB agar containing kanamycin (50 $\left.\mu \mathrm{g} \mathrm{ml}^{-1}\right)$. E. coli BL21 ( $\left.\lambda \mathrm{DE} 3\right)$ cells carrying vector pET28a was used as a control. Both the recombinant and the control were induced with $1 \mathrm{mM}$ IPTG for $2 \mathrm{~h}$. Subsequently, the induced cultures were adjusted to the optical density of 0.1 at $600 \mathrm{~nm}$ and used as inoculum for MIC assays. The concentration of the test drug was diluted twofold in MHB containing kanamycin $\left(50 \mu \mathrm{g} \mathrm{ml}^{-1}\right)$ and IPTG $(1 \mathrm{mM})$. Fifty microliters of inoculum was added to $2 \mathrm{ml}$ of each drug concentration in a 24 -well cell culture plate and incubated at $37^{\circ} \mathrm{C}$ for $18 \mathrm{~h}$ to determine the MIC value as described above. The assays were repeated at least three times.

\section{(iii) MIC assay for $V$. fluvialis isolates in presence of PNA}

MIC of ciprofloxacin was determined for $V$. fluvialis isolates (BD146, L10734, L9978, and L15318) using the same twofold dilution method described above, but in a 96-well polypropylene plate in a total assay volume of $100 \mu \mathrm{l}$ with $5 \mu \mathrm{l}$ of cultures as inoculum. The effect of PNA in reducing the MIC of ciprofloxacin was tested as described previously (Jeon and Zhang, 2009). The assay was initially done with $V$. fluvialis BD146 to check the effect on MIC with increasing concentrations of PNA $(0,2,4$, and $6 \mu \mathrm{M})$. Subsequent to that, assays were performed without and with PNA $(4 \mu \mathrm{M})$ for all the above mentioned $V$. fluvialis isolates.

\section{Mutant Prevention Concentration (MPC) Assay}

Mutant prevention concentration assay was performed as described previously with minor modifications (Marcusson et al., 2005). Hundred microliters of overnight grown culture of each of the $V$. fluvialis isolates (BD146, L15318, L10734, L9978, and L13828) was inoculated in $25 \mathrm{ml} \mathrm{LB}$ and grown at $37^{\circ} \mathrm{C}$ until the optical density at $600 \mathrm{~nm}$ reached $1.0\left(\sim 10^{9}\right.$ cells $\left./ \mathrm{ml}\right)$. The culture was centrifuged at $8000 \mathrm{rpm}$ for $5 \mathrm{~min}$ and the pellet was resuspended in LB to contain $\geq 10^{10}$ cells $/ \mathrm{ml}$. Subsequently, $200 \mu \mathrm{l}$ of resuspended culture was spread on the Muller Hinton Agar (MHA) plates containing defined concentrations of ciprofloxacin. Each strain was tested with six concentrations of ciprofloxacin starting from their MIC (i.e., 1X, 2X, 4X, 8X, 16X, $32 \mathrm{X}$ MIC). The plates were incubated at $37^{\circ} \mathrm{C}$ for $96 \mathrm{~h}$ and MPC was recorded as the lowest concentration of ciprofloxacin that prevented the emergence of mutant colonies. The assays were individually performed three times.

\section{RESULTS}

\section{Presence of qnrVC5 Gene in V. fluvialis Isolates, Characterization of the Gene, and its Flanking Genetic Environment}

The plasmid pBD146 (GenBank accession no. EU574928) obtained from a clinical isolate of $V$. fluvialis BD146, 2002, was earlier reported to harbor a $q n r$ gene (GenBank accession no. JN408080; Rajpara et al., 2009). In another report, the same gene was also found in plasmid preparations from two $V$. fluvialis isolates L10734 (GenBank accession no. JN571550) and L9978 (GenBank accession no. JN571549; Singh et al., 2012) and termed as $q n r V C$-like gene. Later, it was named qnrVC5 allele (Fonseca and Vicente, 2013). The sequence encoded a protein of 218 amino acids with two domains of 11 and 32 pentapeptide repeats bridged by a glycine residue.

Based on the sequence of $\mathrm{pBD} 146$, it was evident that qnrVC5 gene in $V$. fluvialis isolate BD146 was present in the form of a gene cassette where ORF was flanked with a downstream recombination site corresponding to $V$. cholerae repeat (VCR) region and an upstream internal promoter $\left(\mathrm{P}_{\mathrm{qnrVC}}\right)$ with canonical sequence (Figure 1A). Most interestingly, upstream of -10 and -35 sequences of the $\mathrm{P}_{\mathrm{qnrVC}}$ promoter, binding sites for PurR (purine metabolism repressor) and ArgR2 (arginine metabolism regulator) were also predicted in this cassette using Softberry - BPROM online tool (Figure 1A). To further characterize the gene sequence from other two isolates (L10734 and L9978), primers qnrVCcas-F and qnrVCcas-R were designed based on the sequence flanking qnrVC5 in pBD146. PCR carried out using these primers revealed that the same length of gene 


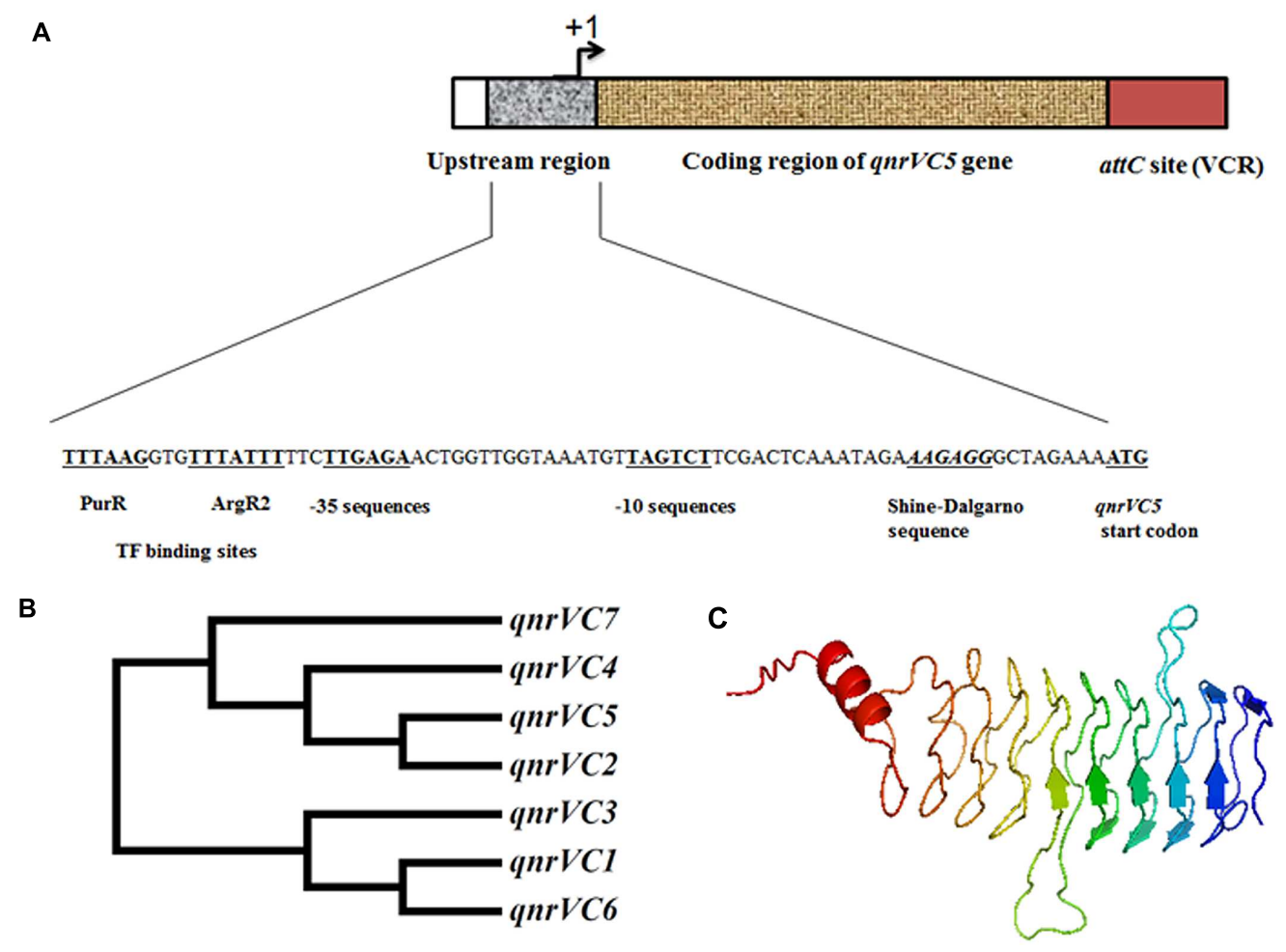

FIGURE 1 | The genetic environment, homology and structure prediction of the gene/gene product of qnrVC5 gene from $\mathbf{V}$. fluvialis isolate BD146. (A) The structure of qnrVC5 gene cassette in pBD146 with canonical promoter sequence and VCR recombination site, at the upstream and downstream positions of the coding region, respectively. (B) Dendrogram of qnrVC5 allele with other qnrVC alleles, showing the close relationship of qnrVC5 with qnrVC2 and qnrVC4. (C) Structure prediction of QnrVC5 using I-TASSER server, showing right handed quadrilateral $\beta$-helix forming 10 coils, interrupted by two loops.

cassette was also amplified from these two isolates suggestive of the similar gene organization in these three bacterial isolates.

\section{Sequence Analysis, Homology, and Structure Prediction for qnrVC5 Gene/Protein}

At nucleotide level, the ORF of qnrVC5 allele shared 99\% identity with qnrVC4 and qnrVC2, and 97\% identity with qnrVC7. $q n r V C 5$ sequence was found to have $76 \%$ identity with qnrVC1 and $75 \%$ with both $q n r V C 3$ and $q n r V C 6$ alleles. The phylogenetic tree for all these $q n r V C$ genes showed that $q n r V C 1, q n r V C 3$, and $q n r V C 6$ belonged to the same group, whereas $q n r V C 2$, qnrVC4, $q n r V C 5$, and $q n r V C 7$ formed another group (Figure 1B). This clearly showed that $q n r V C 5$ was closely related to qnrVC2 and $q n r V C 4$, which corroborated the earlier findings (Fonseca and Vicente, 2013). In addition to this, it was observed that among all the $q n r V C$ alleles listed out by Fonseca and Vicente, (Fonseca and Vicente, 2013) qnrVC1, qnrVC3, and qnrVC6 alleles were associated with $V$. parahaemolyticus repeats (VPRs) whereas qnrVC2, qnrVC4, and qnrVC5 alleles were associated with VCRs, indicating different lineage for these two groups of $q n r V C$ alleles. Three dimensional structure prediction of QnrVC5 protein using I-TASSER server depicted the structure of a typical PRP, threading into $\beta$ helical folds interrupted by two loops (Figure 1C). QnrVC5 protein encompassed 10 coils with two loops (loop A and loop B) extending outward from the regular structure, interrupting the $\beta$-helix turn. Loop A and loop B from QnrVC5 shared 25 and 50\% sequence homology, respectively, with the corresponding loops of QnrB1. Structure predicted from the threading experiments on I-TASSER server was based on the templates of O-methyl transferase (PDB ID: 3DUL) from Bacillus cereus and QnrB1 (PDB ID: 2XTY and 2XTW) from K. pneumoniae. The structural analogs of the predicted structure were QnrB1 (PDB ID: 2XTW), AhQnr (PDB ID: 3PSS) from A. hydrophila and O-methyl transferase (PDB ID: 3DUL).

\section{Contribution of qnrVC5 Gene in Quinolone Resistance of their Native V. fluvialis Host}

The effect of qnrVC5 gene in elevating the MIC and MPC of quinolones in $V$. fluvialis isolates was determined. All the $V$. fluvialis isolates harboring qnrVC5 (BD146, L10734, and L9978) were subjected to MIC assays with nalidixic acid, norfloxacin, ciprofloxacin, and ofloxacin and MPC assays with ciprofloxacin. Among these three strains, L9978 possessed $q n r V C 5$ as the only detected quinolone resistance determinant whereas rest two were having other factors along with qnrVC5 gene (Table 1). V. fluvialis BD146 had GyrA S83I, ParC S85L mutations and $a a c\left(6^{\prime}\right)-I b-c r$ gene as the quinolone resistance factors in addition to qnrVC5 gene. V. fluvialis L10734 had GyrA 
TABLE 1 | Quinolone susceptibility of $V$. fluvialis strains and their corresponding $E$. coli transformants.

\begin{tabular}{|c|c|c|c|c|c|c|}
\hline \multirow[t]{2}{*}{ Strain/transformant } & \multirow{2}{*}{$\begin{array}{l}\text { Quinolone resistance } \\
\text { determinants }\end{array}$} & \multicolumn{4}{|c|}{$\operatorname{MIC}\left(\mu \mathrm{g} \mathrm{ml}^{-1}\right)$} & \multirow{2}{*}{$\begin{array}{c}\text { MPC of Ciprofloxacin } \\
\left(\mu \mathrm{g} \mathrm{ml}^{-1}\right)\end{array}$} \\
\hline & & Nalidixic acid & Norfloxacin & Ciprofloxacin & Ofloxacin & \\
\hline \multicolumn{7}{|l|}{ V. fluvialis } \\
\hline BD146 & $\begin{array}{l}\text { GyrA S83I, ParC S85L, } \\
\text { qnrVC5 and aac }\left(6^{\prime}\right) / b-c r\end{array}$ & 1500 & $17.5-20$ & 10 & 10 & 320 \\
\hline L15318 & GyrA S83I, ParC S85L & 1000 & 15 & $2.5-5$ & 8 & 40 \\
\hline L10734 & $\begin{array}{l}\text { GyrA S83I, ParC S85L and } \\
\text { qnrVC5 }\end{array}$ & 125 & 10 & 2.5 & 8 & 40 \\
\hline L9978 & qnrvC5 & 2 & 1.25 & 0.312 & 0.5 & $1.25-5$ \\
\hline L13828 & None & 0.75 & 0.312 & 0.156 & 0.312 & $0.625-2.5$ \\
\hline \multicolumn{7}{|l|}{ E. coli JM109 } \\
\hline BD146 transformant (7.5 kb+) & qnrVC5 and aac(6') Ib-cr & 400 & 4 & 0.5 & 1 & ND \\
\hline BD146 transformant (7.5 kb-) & $a a c\left(6^{\prime}\right) l b-c r$ & 200 & 2 & 0.25 & 0.5 & ND \\
\hline L10734 transformant & qnrVC5 & 200 & 2 & 0.25 & 1 & ND \\
\hline L9978 transformant & qnrVC5 & 400 & 4 & 0.5 & 2 & ND \\
\hline JM109 (non-transformant) & None & 50 & 1 & 0.125 & 0.5 & ND \\
\hline
\end{tabular}

ND, Not done.

S83I and ParC S85L mutations along with qnrVC5 gene (Table 1). The quinolone resistant V. fluvialis strain L15318 (having GyrA S83I and ParC S85L mutations and lacking qnrVC5) and quinolone sensitive $V$. fluvialis strain L13828 were included as controls (Table 1). The MIC and MPC values of $V$. fluvialis isolates for quinolones have been mentioned in Table 1. It was observed that when compared to the sensitive strain L13828, the qnrVC5-bearing strain L9978 showed about twofold to fourfold elevation in MIC of tested quinolones and twofold elevation in MPC of ciprofloxacin. When compared to L15318, BD146 showed twofold to fourfold increase in MIC of ciprofloxacin and slight increase ( $<$ twofold) in MIC of nalidixic acid, norfloxacin, and ofloxacin presumably due to additional presence of PMQR determinants qnrVC5 gene and aac (6')-Ib-cr gene apart from GyrA and parC mutations. This result also indicated that qnrVC5 and $a a c\left(6^{\prime}\right)-I b-c r$ determinants may chiefly contribute toward resistance to ciprofloxacin. As expected, the MPC of ciprofloxacin was also elevated eightfold in $V$. fluvialis BD146 when compared to L15318 and L10734. Though it may be difficult to compare MIC/MPC of non-isogenic strains, synergy between different resistance factors was evident in majority of cases except L10734 and L15318. Similarly, the lower MIC obtained for nalidixic acid in L10734 as compared to L15318 could not be explained.

\section{Contribution of qnrVC5 Gene in Quinolone Resistance of E. coli Transformants}

In the previous studies from our laboratory, plasmid preparations from V. fluvialis isolates BD146 (Rajpara et al., 2009), L10734, and L9978 (Singh et al., 2012) were transformed into E. coli JM109 to elucidate their transferable traits. E. coli transformants of these three $V$. fluvialis plasmid preparations harboring qnrVC5 gene were utilized in this study to find the effect of this gene in elevating the MIC of quinolones. For BD146, two types of E. coli transformants were observed on the basis of presence or absence of a $7.5 \mathrm{~kb}$ plasmid pBD146 harboring qnrVC5 gene. The transformants that possessed $7.5 \mathrm{~kb}$ plasmid as well as another low copy number plasmid [bearing $a a c\left(6^{\prime}\right) \mathrm{Ib}$-cr gene] were termed as $7.5 \mathrm{~kb}+$. On the other hand, the transformants harboring only low copy number plasmid but devoid of the $7.5 \mathrm{~kb}$ plasmid were termed as $7.5 \mathrm{~kb}$ - (Rajpara et al., 2009). In other words, the transformants $7.5 \mathrm{~kb}+$ possessed both qnrVC5 and $\operatorname{aac}\left(6^{\prime}\right) \mathrm{Ib}$-cr genes whereas $7.5 \mathrm{~kb}$ - transformants were positive for aac (6') Ib-cr gene only (Table 1). Therefore, in the present study, one $7.5 \mathrm{~kb}+$ transformant was selected to study the effect of qnrVC5 gene in combination with aac (6') Ib-cr and one $7.5 \mathrm{~kb}$ transformant was selected to study the effect of $a a c$ (6') Ib-cr alone in quinolone resistance. For similar reasons, transformants of L10734 and L9978 bearing qnrVC5 gene (Singh et al., 2012) were included to study the effect of qnrVC5 gene alone. Untransformed E. coli JM109 was used as a negative control for the MIC assays. The MIC values for all the four transformants and E. coli JM109 are mentioned in Table 1. The transformants having qnrVC5 alone showed twofold to fourfold elevation in MIC of norfloxacin, ciprofloxacin and ofloxacin and fourfold to eightfold increase in the MIC of nalidixic acid when compared to E. coli JM109. The $7.5 \mathrm{~kb}+$ transformant having qnrVC5 along with aac (6') $\mathrm{Ib}$-cr gene showed eightfold increase in MIC of nalidixic acid and fourfold for norfloxacin and ciprofloxacin. The $7.5 \mathrm{~kb}+$ transformant showed twofold increase in the MIC of ofloxacin. The $7.5 \mathrm{~kb}$ - transformant having aac (6') Ib-cr gene alone, showed fourfold increase in MIC for nalidixic acid and twofold for norfloxacin and ciprofloxacin but did not show any effect against ofloxacin.

\section{Expression of qnrVC5 Gene in Native V. fluvialis Host and E. coli Transformants}

RT-PCR was carried out to confirm the expression of qnrVC5 gene and $a a c\left(6^{\prime}\right) \mathrm{Ib}$-cr gene in the native host and their E. coli transformants (Figure 2). In both the cases, genes were expressed 


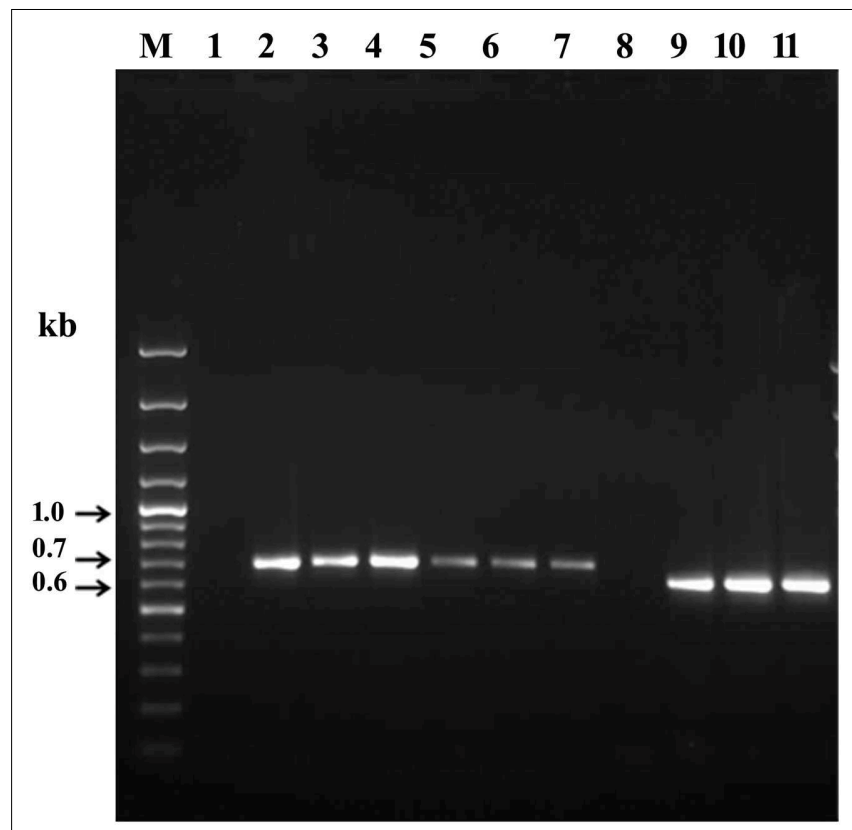

FIGURE 2 | Expression of qnrVC5 and aac(6') lb-cr genes in the native V. fluvialis host and in E. coli JM109 transformants derived from the plasmids of native hosts. $100 \mathrm{bp}$ Gene ruler (Fermentas) was used as the marker (M). Lane 1: Negative control (without reverse transcription step) for RT-PCR product of qnrVC5 transcript (657 bp) from $V$. fluvialis hosts BD146; Lanes 2 to 4: RT-PCR products of qnrVC5 transcript (657 bp) from V. fluvialis hosts BD146, L10734 and L9978, respectively; Lanes 5 to 7: RT-PCR products of qnrVC5 transcript (657 bp) from transformants of BD146 (7.5 kb+), L10734 and L9978; Lane 8: Negative control (without reverse transcription step) for RT-PCR product of aac(6') Ib-cr transcript (657 bp) from V. fluvialis hosts BD146; Lanes 9 to 11: RT-PCR products of aac(6') $\mathrm{Ib}-\mathrm{Cr}$ transcript (608 bp) from $V$. fluvialis BD146 and its transformants $(7.5 \mathrm{~kb}+$ and $7.5 \mathrm{~kb}-)$, respectively.

with the expected band size of $657 \mathrm{bp}$ for qnrVC5 (Figure 2, Lanes 2 to 7) and 608 bp for $a a c\left(6^{\prime}\right) ~ I b-c r$ (Figure 2, Lanes 9 to 11). The negative controls without reverse transcription step that were included to ensure the absence of DNA contamination in RNA templates, did not show any band corresponding to the expressed genes. Two such negative controls with BD146 RNA templates for RT-PCR of $q n r V C 5$ and $a a c$ (6') Ib-cr transcripts have been shown (Figure 2, Lanes 1 and 8, respectively).

\section{Effect of PNA Against qnrVC5 Gene in Native V. fluvialis Host}

Once the presence of $q n r V C 5$ RNA was confirmed in the native host, a peptide-PNA against qnrVC5 RNA was used to silence its expression. MIC of ciprofloxacin for $V$. fluvialis BD146 was determined in the presence of increasing concentrations of PNA $(0,2,4$, and $6 \mu \mathrm{M})$. The results showed a steady decrease of MIC values with increasing concentration of PNA with $4 \mu \mathrm{M}$ of PNA appearing as an effective dose for inhibition. Subsequently, MIC of ciprofloxacin for all the three qnrVC5bearing V. fluvialis isolates (BD146, L10734, and L9978) and one qnrVC5-lacking $V$. fluvialis isolate L15318 was tested at a final concentration of $4 \mu \mathrm{M}$ of PNA. Twofold to eightfold decrease in MIC of ciprofloxacin in all the three isolates was observed in the presence of PNA when compared to the control with no PNA (Table 2). In qnrVC5-lacking V. fluvialis isolate L15318, the MIC of ciprofloxacin was similar irrespective of the presence of PNA indicating that PNA did not have any non-specific inhibitory activity (Table 2).

\section{Gene Cloning and Recombinant Protein Expression}

The qnrVC5 gene was cloned in pET28a expression vector and the authenticity of the recombinant clones was confirmed. A protein band of $\sim 27 \mathrm{kDa}$ was found to be overexpressed by the recombinants on $1 \mathrm{mM}$ IPTG induction. This protein band was excised from the SDS-PAGE gel and subjected to trypsin digestion. The peptide mass fingerprinting analysis confirmed it to be QnrVC5.

\section{Elevation of MIC for quinolones in qnrVC5 Recombinants}

Minimum inhibitory concentration assays were carried out to study the functionality of recombinant QnrVC5 protein. MIC was tested with different generations of quinolones with nalidixic acid representing first generation, norfloxacin, ciprofloxacin, ofloxacin, and levofloxacin representing second generation, sparfloxacin for third generation and moxifloxacin as a representative of fourth generation. Interestingly, recombinant $E$. coli BL21( $\lambda \mathrm{DE} 3)$ cells were found to show eightfold to more than 64-fold increase in MIC of different quinolones when compared to control having pET28a alone (Table 3). QnrVC5 conferred higher resistance toward ciprofloxacin and sparfloxacin as the MICs of these two drugs were elevated to more than 64-fold. MIC of levofloxacin and moxifloxacin were elevated to 64-fold and 32-fold, respectively. QnrVC5 elevated the MIC of both norfloxacin and ofloxacin to 16 -fold and nalidixic acid to eightfold (Table 3).

\section{DISCUSSION}

In the studies aimed at unraveling the molecular mechanisms of drug resistance in the clinical isolates of $V$. fluvialis, a new allele named qnrVC5 was reported from this laboratory (Rajpara et al., 2009; Singh et al., 2012). The current study was intended to characterize this plasmid-associated gene from those clinical isolates, for its role in conferring protection/resistance toward quinolones. qnrVC5 ORF was found as a gene cassette with its

TABLE 2 | Silencing effect of qnrVC5 gene in V. fluvialis strains.

\begin{tabular}{lccc}
\hline V. fluvialis & MIC of Ciprofloxacin $\left(\boldsymbol{\mu} \mathbf{~ m ~}^{-\mathbf{1}}\right)$ & \multirow{2}{*}{ Fold in reduction of MIC } \\
\cline { 2 - 3 } & PNA- & PNA+ & \\
\hline BD146 & $10-20$ & $2.5-5$ & $2-8$ \\
L10734 & 2.5 & 1.25 & 2 \\
L9978 & 0.156 & 0.078 & 2 \\
L15318 & 5 & 5 & 0
\end{tabular}


TABLE 3 | MIC of quinolones for pET-qnrVC5 clone.

\begin{tabular}{|c|c|c|c|}
\hline \multirow[t]{2}{*}{ Quinolones } & \multicolumn{2}{|c|}{$\operatorname{MIC}\left(\mu \mathrm{g} \mathrm{ml}^{-1}\right)$} & \multirow[t]{2}{*}{ Fold in elevation of MIC } \\
\hline & E. coli (BL21 גDE3) (pET-qnrVC5 clone) & E. coli (BL21 גDE3) (pET28a) & \\
\hline Nalidixic acid & 5 & 0.625 & 8 \\
\hline Norfloxacin & 0.125 & 0.0078 & 16 \\
\hline Ciprofloxacin & 0.0156 & $<0.000243$ & $>64$ \\
\hline Ofloxacin & 0.0625 & 0.0039 & 16 \\
\hline Levofloxacin & 0.0312 & 0.000487 & 64 \\
\hline Sparfloxacin & 0.125 & $<0.00195$ & $>64$ \\
\hline Moxifloxacin & 0.125 & 0.0039 & 32 \\
\hline
\end{tabular}

own promoter with canonical sequence and attC site which was identical to $V$. cholerae repeats (VCR). BLAST search indicated that this gene cassette showed $99 \%$ identity with qnrVC2 gene cassette found in the plasmid pVN84 harbored by $V$. cholerae O1, isolated from Vietnam during 2004 as well as qnrVC5 gene cassette found in another plasmid present in V. parahaemolyticus V110, isolated from Hong Kong during 2010. The qnrVC5 gene cassette also showed $99 \%$ identity to qnrVC4 gene cassettes carried in class I integrons of Aeromonas caviae, A. hydrophila, E. coli, and Salmonella enterica and $98 \%$ identity to qnrVC4 gene in class I integron of $A$. punctata. This indicated evolutionary relationship among $q n r V C 2, q n r V C 5$, and $q n r V C 4$ gene cassettes and their dissemination in various species of bacteria through mobile genetic elements. qnrVC5 gene cassette showed 97\% homology with a $q n r$ cassette found in super integron (SI) on the small chromosome of $V$. cholerae MS6 (Okada et al., 2014). The VCR region downstream of qnrVC5 ORF, showed identity to the stretch of sequences present in the chromosome of various Vibrio and Shewanella species. This indicated the possible exchange of various resistance conferring genes or gene cassettes among these bacterial species of Shewanellaceae and Vibrionaceae families in their environmental vicinity. This observation supported the hypothesis by Poirel et al. (2005a,b) that these two families could be the reservoirs of $q n r$ genes. This additionally supported the chromosomal origin of $q n r$ alleles. The presence of regulatory motif for DNA-binding proteins PurR and ArgR indicated the possible involvement of these elements in controlling the expression of qnrVC5 gene. This was suggestive of the probable biological function of Qnr proteins in relation to purine and arginine metabolism or indicate that qnrVC5 might be one of the potentially co-regulated set of genes interlinked with amino acid and nucleotide metabolism.

The antimicrobial susceptibility assays clearly showed the involvement of $q n r V C 5$ gene in conferring resistance to different quinolones. Compared to the sensitive $V$. fluvialis strain (L13828), qnrVC5-bearing-isolates resisted the quinolone action by virtue of $q n r V C 5$ alone as in L9978 or in unison with other mechanisms as in BD146 and L10734. The qnrVC5- bearingplasmids from the parent strain, transformed to the heterologous host E. coli JM109, proved the role played by the gene in reducing susceptibility toward quinolones. Hence, the qnrVC5 gene could effectively express its traits in different bacterial hosts as these plasmids disseminated through horizontal gene transfer.
Silencing of the gene increased the susceptibility of the parent strains toward ciprofloxacin from twofold to eightfold, again confirming the role of qnrVC5 in drug resistance.

The results of MIC and MPC assays were vivid representations of synergy between various quinolone resistance determinants (with some exceptions) with BD146 as a carrier of all the three determinants and rest of the isolates as carriers of either one or two determinants. These results also clearly indicated the major role of topoisomerase mutations in susceptibility for quinolones with qnrVC5 or aac (6) $\mathrm{Ib}-\mathrm{cr}$, playing the role of an apprentice. Though the level of resistance conferred by qnrVC5 for ciprofloxacin was low, it apparently helped the pathogen in elevating the MIC and MPC of ciprofloxacin for $V$. fluvialis strains (BD146 and L9978) thus extending the mutant selection window for ciprofloxacin in these strains. This is likely to help in the selection of more mutants at higher concentrations of drug, by enhancing the survival ability of the pathogen (Strahilevitz et al., 2009). However, as described in the results section, there were anomalies in this synergistic design and therefore could not be explained. Perhaps, comparisons of susceptibility trends in non-isogenic strains may not be very straight-forward and may reflect a complex interplay of many known and yet unknown genetic factors.

Recently, the role of $q n r V C 1$ gene located in a chromosomal integron in clinical and environmental Pseudomonas aeruginosa isolates, in conferring resistance to quinolones, has been described (Belotti et al., 2015). qnrVC1 gene with its promoter was cloned in E. coli and $P$. aeruginosa and shown to confer variable resistance to quinolones. In the present study, T7 promoter-based overexpression of $q n r V C 5$ gene was utilized to study the gene function in isolation which was not possible in native $V$. fluvialis isolates. MIC study with the recombinant reflected that the potency of this gene in conferring resistance is significant and variable resistance was observed for different generations of quinolones.

Using I-TASSER server, structure of QnrVC5 protein was predicted and was found to be similar to that of QnrB1 (Vetting et al., 2011) and AhQnr structure (Xiong et al., 2011) implying that the function of QnrVC5 could also be similar to the above two proteins (i.e., imparting quinolone resistance). The important feature in the structures of QnrB1 and AhQnr is the presence of two loops and their contribution in protection of DNA gyrase from quinolones 
(Vetting et al., 2011; Xiong et al., 2011). Therefore, the working model of Qnr proteins (PRPs with loops) in quinolone resistance was proposed, in which the Qnr proteins disturb the quinolone-topoisomerase-DNA complex (Vetting et al., 2011; Xiong et al., 2011). Our functional analysis of recombinant QnrVC5 protein also strengthened the above mentioned model. QnrVC5 protein was shown to elevate the MIC of different quinolones by varying degrees. The possible reason for varied elevation in MIC would be the consequence of varying binding orientations of the different quinolones with the topoisomerase-DNA complex. For example, the interactions of nalidixic acid and ciprofloxacin with gyrase differ in drug binding orientation (perhaps reflected in differences in their potency) such that Qnr destabilizes the ciprofloxacin interaction to a greater extent than the nalidixic acid interaction.

\section{CONCLUSION}

These findings prove that the carriage of $q n r V C$ alleles on various mobile genetic elements such as plasmids, integrons, and SXT elements in a variety of organisms of different genera have been disseminating quinolone resistance widely throughout the globe (Fonseca et al., 2008; Rajpara et al., 2009; Kim et al., 2010; Kumar and Thomas, 2011; Fonseca and Vicente, 2013). With the indiscriminate use of quinolones, this can turn out to be both a reason as well as a consequence of the serious problem of multi-drug resistance.

\section{REFERENCES}

Baranwal, S., Dey, K., Ramamurthy, T., Nair, G. B., and Kundu, M. (2002). Role of active efflux in association with target gene mutations in fluoroquinolone resistance in clinical isolates of Vibrio cholerae. Antimicrob. Agents Chemother. 46, 2676-2678. doi: 10.1128/AAC.46.8.2676-2678.2002

Belotti, P. T., Thabet, L., Laffargue, A., Andre, C., Coulange-Mayonnove, L., Arpin, C., et al. (2015). Description of an integron encompassing blaVIM-2, qnrVC1 and genes encoding bacterial group II intron proteins in Pseudomonas aeruginosa. J. Antimicrob. Chemother. 70, 2237-2240. doi: 10.1093/jac/d kv103

Bhardwaj, A. K. (2015). "Multi-drug resisance in vibrios: never underestimate bacteria," in Advances in Biotechnology and Allied Areas, eds N. N. Nawani, M. Khetmalas, P. M. Razdan, A. Pandey, and K. M. Powar (New Delhi: I. K. International Publishing House Pvt. Ltd.), 91-105.

Bhardwaj, A. K., Vinothkumar, K., Rajpara, N., Mohanty, P., and Kutar, B. M. R. N. S. (2014). "Therapeutic limitations due to antibiotic drug resistance: road to alternate therapies," in Frontiers in Anti-Infective Drug Discovery, Vol. 3, eds A. Rahman and M. I. Choudhary (Sharjah: Bentham Science Publiser), 72-141.

Bhattacharjee, S., Bal, B., Pal, R., Niyogi, S. K., and Sarkar, K. (2010). Is Vibrio fluvialis emerging as a pathogen with epidemic potential in coastal region of eastern India following cyclone Aila? J. Health Popul. Nutr. 28, 311-317.

Fonseca, E. L., Freitas, F., Vieira, V. V., and Vicente, A. C. (2008). New qnr gene cassettes associated with superintegron repeats in Vibrio cholerae O1. Emerg. Infect. Dis. 14, 1129-1131. doi: 10.3201/eid1407.080132

Fonseca, E. L., and Vicente, A. C. (2013). Epidemiology of qnrVC alleles and emergence out of the Vibrionaceae family. J. Med. Microbiol. 62, 1628-1630. doi: 10.1099/jmm.0.062661-0

Good, L., Awasthi, S. K., Dryselius, R., Larsson, O., and Nielsen, P. E. (2001). Bactericidal antisense effects of peptide-PNA conjugates. Nat. Biotechnol. 19, 360-364. doi: 10.1038/86753

\section{AUTHORS CONTRIBUTIONS}

Conceived and designed the experiments: AB and KV. Performed the experiments: KV. Analyzed the data: AB, GK, and KV. Contributed reagents/materials/analysis tools: $\mathrm{AB}$. Wrote the paper: $\mathrm{AB}, \mathrm{GK}$, and $\mathrm{KV}$.

\section{FUNDING}

This work was supported by the grants from Gujarat State Biotechnology Mission (GSBTM; No. GSBTM/MD/PROJECTS/ SSA/3386/2012-13), Department of Biotechnology (DBT), Ministry of Science and Technology, Government of India (BT/PR/11634/INF/22/104/2008) and Indian Council of Medical Research (ICMR; No.80/845/2013-ECD-I). Authors thankfully acknowledge The Puri Foundation for Education in India for providing infrastructure facilities. KV is the recipient of Senior Research Fellowship from ICMR grant mentioned above.

\section{ACKNOWLEDGMENTS}

The authors are grateful to Dr. Amit Ghosh, Dr. T. Ramamurthy and Dr. S. K. Niyogi, National Institute of Cholera and Enteric Diseases (NICED), Kolkata, India, for their support and advice. The authors thank Ms. Neha Rajpara, Mr. Priyabrata Mohanty, Mr. Braj M. N. R. S. Kutar, Ms. Aneri Shah, and Mr. Shailesh Bhalara for their suggestions and technical support.

Hegde, S. S., Vetting, M. W., Mitchenall, L. A., Maxwell, A., and Blanchard, J. S. (2011). Structural and biochemical analysis of the pentapeptide repeat protein EfsQnr, a potent DNA gyrase inhibitor. Antimicrob. Agents Chemother. 55, 110-117. doi: 10.1128/AAC.01158-10

Hegde, S. S., Vetting, M. W., Roderick, S. L., Mitchenall, L. A., Maxwell, A., Takiff, H. E., et al. (2005). A fluoroquinolone resistance protein from Mycobacterium tuberculosis that mimics DNA. Science 308, 1480-1483. doi: 10.1126/science.1110699

Jeon, B., and Zhang, Q. (2009). Sensitization of Campylobacter jejuni to fluoroquinolone and macrolide antibiotics by antisense inhibition of the CmeABC multidrug efflux transporter. J. Antimicrob. Chemother. 63, 946-948. doi: 10.1093/jac/dkp067

Jeong, J. Y., Kim, E. S., Choi, S. H., Kwon, H. H., Lee, S. R., Lee, S. O., et al. (2008) Effects of a plasmid-encoded qnrAl determinant in Escherichia coli strains carrying chromosomal mutations in the acrAB efflux pump genes. Diagn. Microbiol. Infect. Dis. 60, 105-107. doi: 10.1016/j.diagmicrobio.2007.07.015

Kim, E. S., and Hooper, D. C. (2014). Clinical importance and epidemiology of quinolone resistance. Infect. Chemother. 46, 226-238. doi: 10.3947/ic.2014.46.4.226

Kim, H. B., Wang, M., Ahmed, S., Park, C. H., Larocque, R. C., Faruque, A. S., et al. (2010). Transferable quinolone resistance in Vibrio cholerae. Antimicrob. Agents Chemother. 54, 799-803. doi: 10.1128/AAC. 01045-09

Kumar, P., and Thomas, S. (2011). Presence of qnrVC3 gene cassette in SXT and class 1 integrons of Vibrio cholerae. Int. J. Antimicrob. Agents 37, 280-281. doi: 10.1016/j.ijantimicag.2010.12.006

Liu, M., and Chen, S. (2013). Draft genome sequence of vibrio parahaemolyticus V110, Isolated from Shrimp in Hong Kong. Genome Announc. 1:e300-e313. doi: 10.1128/genomeA.00300-13

Marcusson, L. L., Olofsson, S. K., Komp Lindgren, P., Cars, O., and Hughes, D. (2005). Mutant prevention concentrations of ciprofloxacin for urinary tract 
infection isolates of Escherichia coli. J. Antimicrob. Chemother. 55, 938-943. doi: 10.1093/jac/dki136

Martinez-Martinez, L., Pascual, A., Garcia, I., Tran, J., and Jacoby, G. A. (2003). Interaction of plasmid and host quinolone resistance. J. Antimicrob. Chemother. 51, 1037-1039. doi: 10.1093/jac/dkg157

Martinez-Martinez, L., Pascual, A., and Jacoby, G. A. (1998). Quinolone resistance from a transferable plasmid. Lancet 351, 797-799. doi: 10.1016/S01406736(97)07322-4

Mohanty, P., Patel, A., and Kushwaha Bhardwaj, A. (2012). Role of H- and DMATE-type transporters from multidrug resistant clinical isolates of Vibrio fluvialis in conferring fluoroquinolone resistance. PLoS ONE 7:e35752. doi: 10.1371/journal.pone.0035752

Okada, K., Na-Ubol, M., Natakuathung, W., Roobthaisong, A., Maruyama, F., Nakagawa, I., et al. (2014). Comparative genomic characterization of a Thailand-Myanmar isolate, MS6, of Vibrio cholerae O1 El Tor, which is phylogenetically related to a "US Gulf Coast" clone. PLoS ONE 9:e98120. doi: 10.1371/journal.pone.0098120

Poirel, L., Liard, A., Rodriguez-Martinez, J. M., and Nordmann, P. (2005a). Vibrionaceae as a possible source of Qnr-like quinolone resistance determinants. J. Antimicrob. Chemother. 56, 1118-1121. doi: 10.1093/jac/dki371

Poirel, L., Rodriguez-Martinez, J. M., Mammeri, H., Liard, A., and Nordmann, P. (2005b). Origin of plasmid-mediated quinolone resistance determinant QnrA. Antimicrob. Agents Chemother. 49, 3523-3525. doi: 10.1128/AAC.49.8.35233525.2005

Pons, M. J., Gomes, C., and Ruiz, J. (2013). QnrVC, a new transferable Qnr-like family. Enferm. Infecc. Microbiol. Clin. 31, 191-192. doi: 10.1016/j.eimc.2012.09.008

Rajpara, N., Patel, A., Tiwari, N., Bahuguna, J., Antony, A., Choudhury, I., et al. (2009). Mechanism of drug resistance in a clinical isolate of Vibrio fluvialis: involvement of multiple plasmids and integrons. Int. J. Antimicrob. Agents 34, 220-225. doi: 10.1016/j.ijantimicag.2009.03.020

Ramamurthy, T., Chowdhury, G., Pazhani, G. P., and Shinoda, S. (2014). Vibrio fluvialis: an emerging human pathogen. Front. Microbiol. 5:91. doi: 10.3389/fmicb.2014.00091

Roy, A., Kucukural, A., and Zhang, Y. (2010). I-TASSER: a unified platform for automated protein structure and function prediction. Nat. Protoc. 5, 725-738. doi: 10.1038/nprot.2010.5

Roy, A., Yang, J., and Zhang, Y. (2012). COFACTOR: an accurate comparative algorithm for structure-based protein function annotation. Nucleic Acids Res. 40, W471-W477. doi: 10.1093/nar/gks372

Rushdy, A. A., Mabrouk, M. I., Abu-Sef, F. A., Kheiralla, Z. H., Mohamed AbdelAll, S., and Saleh, N. M. (2013). Contribution of different mechanisms to the resistance to fluoroquinolones in clinical isolates of Salmonella enterica. Braz. J. Infect. Dis. 17, 431-437. doi: 10.1016/j.bjid.2012.11.012

Saga, T., Kaku, M., Onodera, Y., Yamachika, S., Sato, K., and Takase, H. (2005). Vibrio parahaemolyticus chromosomal qnr homologue VPA0095: demonstration by transformation with a mutated gene of its potential to reduce quinolone susceptibility in Escherichia coli. Antimicrob. Agents Chemother. 49, 2144-2145. doi: 10.1128/AAC.49.5.2144-2145.2005

Sanchez, M. B., Hernandez, A., Rodriguez-Martinez, J. M., Martinez-Martinez, L., and Martinez, J. L. (2008). Predictive analysis of transmissible quinolone resistance indicates Stenotrophomonas maltophilia as a potential source of a novel family of Qnr determinants. BMC Microbiol. 8:148. doi: 10.1186/14712180-8-148

Singh, R., Rajpara, N., Tak, J., Patel, A., Mohanty, P., Vinothkumar, K., et al. (2012). Clinical isolates of Vibrio fluvialis from Kolkata, India, obtained during 2006: plasmids, the qnr gene and a mutation in gyrase A as mechanisms of multidrug resistance. J. Med. Microbiol. 61, 369-374. doi: 10.1099/jmm.0.03 7226-0

Srinivasan, V. B., Virk, R. K., Kaundal, A., Chakraborty, R., Datta, B., Ramamurthy, T., et al. (2006). Mechanism of drug resistance in clonally related clinical isolates of Vibrio fluvialis isolated in Kolkata, India. Antimicrob. Agents Chemother. 50, 2428-2432. doi: 10.1128/AAC.01561-05

Strahilevitz, J., Jacoby, G. A., Hooper, D. C., and Robicsek, A. (2009). Plasmidmediated quinolone resistance: a multifaceted threat. Clin. Microbiol. Rev. 22, 664-689. doi: 10.1128/CMR.00016-09

Tamura, K., Stecher, G., Peterson, D., Filipski, A., and Kumar, S. (2013). MEGA6: molecular evolutionary genetics analysis version 6.0. Mol. Biol. Evol. 30, 27252729. doi: 10.1093/molbev/mst197

Thungapathra, M., Sinha, K. K., Chaudhuri, S. R., Garg, P., Ramamurthy, T., Nair, G. B., et al. (2002). Occurrence of antibiotic resistance gene cassettes aac(6')Ib, dfrA5, dfrA12, and ereA2 in class I integrons in non-O1, non-O139 Vibrio cholerae strains in India. Antimicrob. Agents Chemother. 46, 2948-2955. doi: 10.1128/AAC.46.9.2948-2955.2002

Tran, J. H., and Jacoby, G. A. (2002). Mechanism of plasmid-mediated quinolone resistance. Proc. Natl. Acad. Sci. U.S.A. 99, 5638-5642. doi: 10.1073/pnas.082092899

Vetting, M. W., Hegde, S. S., Wang, M., Jacoby, G. A., Hooper, D. C., and Blanchard, J. S. (2011). Structure of QnrB1, a plasmid-mediated fluoroquinolone resistance factor. J. Biol. Chem. 286, 25265-25273. doi: 10.1074/jbc.M111.226936

Xiong, X., Bromley, E. H., Oelschlaeger, P., Woolfson, D. N., and Spencer, J. (2011). Structural insights into quinolone antibiotic resistance mediated by pentapeptide repeat proteins: conserved surface loops direct the activity of a Qnr protein from a gram-negative bacterium. Nucleic Acids Res. 39, 3917-3927. doi: $10.1093 /$ nar/gkq1296

Zhang, Y. (2008). I-TASSER server for protein 3D structure prediction. BMC Bioinformatics 9:40. doi: 10.1186/1471-2105-9-40

Zhu, J. M., Jiang, R. J., Kong, H. S., Zhang, R., Lu, H. X., Sun, C. G., et al. (2013). [Emergence of novel variants of gyrA, parC, qnrS genes in multi-drug resistant Klebsiella caused pneumonia]. Zhonghua Liu Xing Bing Xue Za Zhi. 34, 61-66.

Conflict of Interest Statement: The authors declare that the research was conducted in the absence of any commercial or financial relationships that could be construed as a potential conflict of interest.

Copyright (c) 2016 Vinothkumar, Kumar and Bhardwaj. This is an open-access article distributed under the terms of the Creative Commons Attribution License (CC BY). The use, distribution or reproduction in other forums is permitted, provided the original author(s) or licensor are credited and that the original publication in this journal is cited, in accordance with accepted academic practice. No use, distribution or reproduction is permitted which does not comply with these terms. 